\title{
Self-assembly and conformation of tetrapyridyl-porphyrin molecules on $\mathrm{Ag}(111)$
}

\author{
W. Auwärter, ${ }^{\text {a) }}$ A. Weber-Bargioni, A. Riemann, ${ }^{\text {b) }}$ and A. Schiffrin \\ Department of Chemistry and Physics \& Astronomy, University of British Columbia, Vancouver, \\ British Columbia V6T 1Z4, Canada \\ O. Gröning and R. Fasel \\ Swiss Federal Laboratories for Materials Testing and Research (EMPA), 3602 Thun, Switzerland \\ J. V. Barth \\ Department of Chemistry and Physics \& Astronomy, University of British Columbia, Vancouver, \\ British Columbia V6T 1Z4, Canada
}

(Received 21 December 2005; accepted 16 March 2006; published online 17 May 2006)

\begin{abstract}
We present a low-temperature scanning tunneling microscopy (STM) study on the supramolecular ordering of tetrapyridyl-porphyrin (TPyP) molecules on $\operatorname{Ag}(111)$. Vapor deposition in a wide substrate temperature range reveals that TPyP molecules easily diffuse and self-assemble into large, highly ordered chiral domains. We identify two mirror-symmetric unit cells, each containing two differently oriented molecules. From an analysis of the respective arrangement it is concluded that lateral intermolecular interactions control the packing of the layer, while its orientation is induced by the coupling to the substrate. This finding is corroborated by molecular mechanics calculations. High-resolution STM images recorded at $15 \mathrm{~K}$ allow a direct identification of intramolecular features. This makes it possible to determine the molecular conformation of TPyP on $\operatorname{Ag}(111)$. The pyridyl groups are alternately rotated out of the porphyrin plane by an angle of $60^{\circ}$. () 2006 American Institute of Physics. [DOI: 10.1063/1.2194541]
\end{abstract}

\section{INTRODUCTION}

The controlled assembly of highly organized architectures on surfaces using functional molecular species is a promising approach to design novel nanostructured materials. Notably the formation and characterization of porphyrin adlayers on surfaces are of great interest from the fundamental and technological points of view. This class of molecules plays an important role in biological processes such as oxygen transport and photosynthesis. Accordingly, thin films assembled from these versatile molecular building blocks can be applied as optical switches, ${ }^{1}$ solar cells, ${ }^{2,3}$ and sensors. ${ }^{4}$

In the last decade two-dimensional assemblies of mesosubstituted porphyrins have been investigated on various substrates making use of the excellent real space imaging capabilities of scanning tunneling microscopy (STM). Particular attention was given to two issues that embody the functionality of this class of porphyrins. First, the mesosubstituents, often referred to as legs, are able to rotate around the $\mathrm{C}-\mathrm{C} \sigma$ bond connecting each of them to the porphyrin core (cf. Fig. 1). This allows conformational adaptation of the molecule to its local environment. Accordingly, a wide range of dihedral angles $\Theta$ is observed. While large values $\left(60^{\circ} \leqslant \Theta \leqslant 90^{\circ}\right)$ are typical in solid phases ${ }^{5-7}$ smaller angles down to $\Theta=0^{\circ}$ are suggested for molecules adsorbed on metal surfaces, ${ }^{8,9}$ promoted by the increased coupling of the molecular $\pi$ system to the substrate. The rotation of a leg can

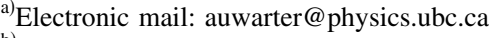

${ }^{b)}$ Present address: Department of Physics and Astronomy, Western Washington University, Bellingham, Washington 98225, USA
}

also be directly induced by a tip of a scanning probe microscope, ${ }^{10}$ which even allows to determine the energy necessary to operate such a molecular "switch." ${ }^{, 11}$ However, dihedral angles below roughly $60^{\circ}$ result in distortions of the initially planar porphyrin core, ${ }^{12}$ as steric repulsion between hydrogen atoms of the ring and the hydrogens of the porphyrin macrocycle sets in (c.f., Refs. 13 and 14). Second, mesosubstituted porphyrins with a wide variety of end-groups can be synthesized. This makes them well-established building blocks for metal-organic networks in solid state chemistry. ${ }^{15}$

On surfaces their adsorption and self-assembly behavior in dependence on the chemical characteristics of the substituents is of special interest. It is the subtle balance between adsorbate-adsorbate and adsorbate-substrate interactions that controls the self-assembly of low-dimensional supramolecular nanostructures. For example, porphyrins functionalized by carboxyl groups enabling hydrogen bonding show an intermolecular ordering very different from the close-packed arrangement expected without hydrogen bonds. ${ }^{16}$ Alternatively, substituents with an asymmetric charge distribution promoting dipole-dipole interactions between the porphyrins allows to assemble supramolecular aggregates with controlled size and shape, while the original porphyrins form compact islands. ${ }^{13,17}$

The influence of the substrate on the porphyrin adlayer formation process and the resulting structure was studied in solution on graphite and various $\mathrm{Au}$ surfaces. ${ }^{18-24}$ Additionally, self-assembly of vapor deposited porphyrins was explored on several $\mathrm{Au}, \mathrm{Ag}$, and $\mathrm{Cu}$ surfaces. ${ }^{25-30}$

Here, we focus for the first time on the supramolecular 

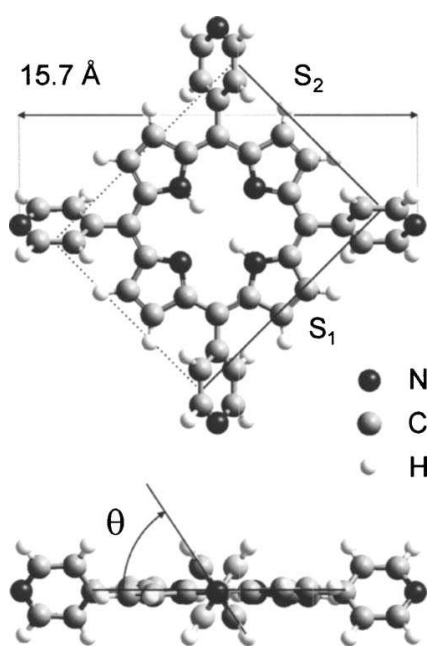

FIG. 1. Model of the tetrapyridyl-porphyrin (TPyP) molecule in its groundstate conformation. The top view (upper panel) highlights the central porphyrin core and the four terminal pyridyl groups, the legs. The latter are alternately rotated out of the porphyrin macrocycle plane resulting in two different sidelengths $S_{1}$ and $S_{2}$ in the STM data. In the side view (lower panel) the dihedral angle $\Theta \sim 60^{\circ}$ between the porphyrin plane and the pyridyl rings is indicated.

ordering of a vapor deposited pyridil terminated porphyrin species in two dimensions (2D). Specifically, we used 5,10,15,20-tetra(4-pyridyl)porphyrin (TPyP) molecules on $\mathrm{Ag}(111)$. TPyP, as shown in Fig. 1, consists of a central porphyrin macrocycle and four terminal pyridyl rings. The $N$ termination of the lateral groups, that favors linking to metal centers, makes TPyP a promising building block for supramolecular aggregates ${ }^{31,32}$ and elaborated metal-organic architectures. ${ }^{33-36}$ Our high-resolution images of TPyP reveal well-ordered two-dimensional layers on $\mathrm{Ag}(111)$. From the data we determine the molecular conformation of the adsorbed species on the surface and conclude that the packing of the layer is controlled by intermolecular interactions while the orientation of the layer is induced by the coupling to the $\operatorname{Ag}(111)$ substrate.

\section{EXPERIMENTAL SECTION}

All experiments were performed in a custom-designed ultrahigh vacuum (UHV) apparatus with a base pressure below $2 \times 10^{-10}$ mbar, comprising a low-temperature scanning tunneling microscope (LT-STM). This highly stable instrument $^{37}$ capable of atomic manipulation was designed by G. Meyer at FU Berlin and is commercially available through Createc. ${ }^{38}$ The $\mathrm{Ag}(111)$ single crystal surface was cleaned by repeated cycles of $800 \mathrm{eV} \mathrm{Ar}^{+}$sputtering followed by annealing to $750 \mathrm{~K}$. Subsequently, TPyP $(97+\%$ purity, Frontier Scientific) was deposited by organic molecular beam epitaxy (OMBE) from a quartz crucible held at $525 \mathrm{~K}$. Typical evaporation rates are roughly $0.03 \mathrm{Ml} / \mathrm{min}$. The TPyP was thoroughly degassed prior to any experiments resulting in a background pressure in the $10^{-10}$ mbar range during deposition. Typically, coverages below full monolayer saturation were employed for the TPyP layers, in order to have bare metal patches available to form the STM tip and to determine the apparent height of the TPyP molecules. After dosing TPyP at substrate temperatures in the range of
100-500 K, the sample was cooled down and transferred into the STM, where constant current images were recorded at $\sim 15 \mathrm{~K}$ using electrochemically etched tungsten tips.

In agreement with earlier STM studies and investigations employing complementary experimental techniques sensitive to the chemical structure of the molecules as photoemission and $\mathrm{x}$-ray absorption we presume that vapor deposited porphyrins adsorb intact on the sample surface. ${ }^{39,40}$ Furthermore, it was shown that tunneling on porphyrin adlayers applying resistances typical for molecular imaging does not distort the molecular electronic structure. ${ }^{41}$

\section{RESULTS AND DISCUSSION}

\section{A. Two-dimensional self-assembly}

Figure 2 shows the STM images of the highly ordered layer structure resulting from TPyP deposition on $\mathrm{Ag}(111)$. The formation of large domains extended over hundreds of nanometers even at room temperature indicates a low diffusion barrier of TPyP on $\mathrm{Ag}(111)$. Accordingly, no single molecules can be immobilized even by lowering the substrate temperature to $100 \mathrm{~K}$ during deposition. One observes a staggered arrangement of clearly resolved molecular units. All molecules within a row (I) following a given direction are oriented the same way, while this orientation switches in the neighboring rows (II). Consequently, every second row shows the same molecular orientation. This characteristic pattern evolves in a wide substrate temperature range of 300-500 K. Figure 2(c) highlights the intramolecular features already detectable on the rather large scales of Figs. 2(a) and 2(b). Besides the core with a depression in the center, one can identify four protrusions forming a rectangle. From the molecular dimensions we assign each protrusion to one of the pyridyl legs. This is in agreement with the generally encountered parallel adsorption geometry of the porphyrin macrocycle on metal surfaces and indicates that the pyridyl groups are not oriented perpendicular to the molecular core (dihedral angle $\Theta \neq 90^{\circ}$ ). The corresponding models in Fig. 2(c) clarify the structure and help to identify molecular features.

We describe this TPyP structure by a nearly rectangular unit cell with a molecule in every corner and a central molecule in a different azimuthal orientation. The unit cell parameters are as follows: $b_{1}=13.9 \pm 0.2 \AA, b_{2}=27.4 \pm 0.2 \AA$, and $\beta=93 \pm 2^{\circ}$.

On the $\operatorname{Ag}(111)$ surface one can identify two types of domains, labeled $D_{\delta}$ and $D_{\lambda}$, respectively. They are drawn schematically in Fig. 3. The axis of the corner molecules is rotated either clockwise $\left(D_{\delta}\right)$ or counterclockwise $\left(D_{\lambda}\right)$ away from the long side of the unit cell. Thus, the nonchiral TPyP building blocks assemble into chiral domains, i.e., there is enantiomorphic ordering. ${ }^{42-44}$ When checking the azimuthal orientation of the two domains relative to the substrate, we find that the domains are rotated by an angle $\pm \alpha=16^{\circ}$ away from the close-packed substrate rows. One domain clockwise $(+)$, the other one counterclockwise $(-)$. This means that these two domains are mirror images of each other, with the mirror plane perpendicular to the surface aligned along a substrate high symmetry direction. Consequently, the do- 

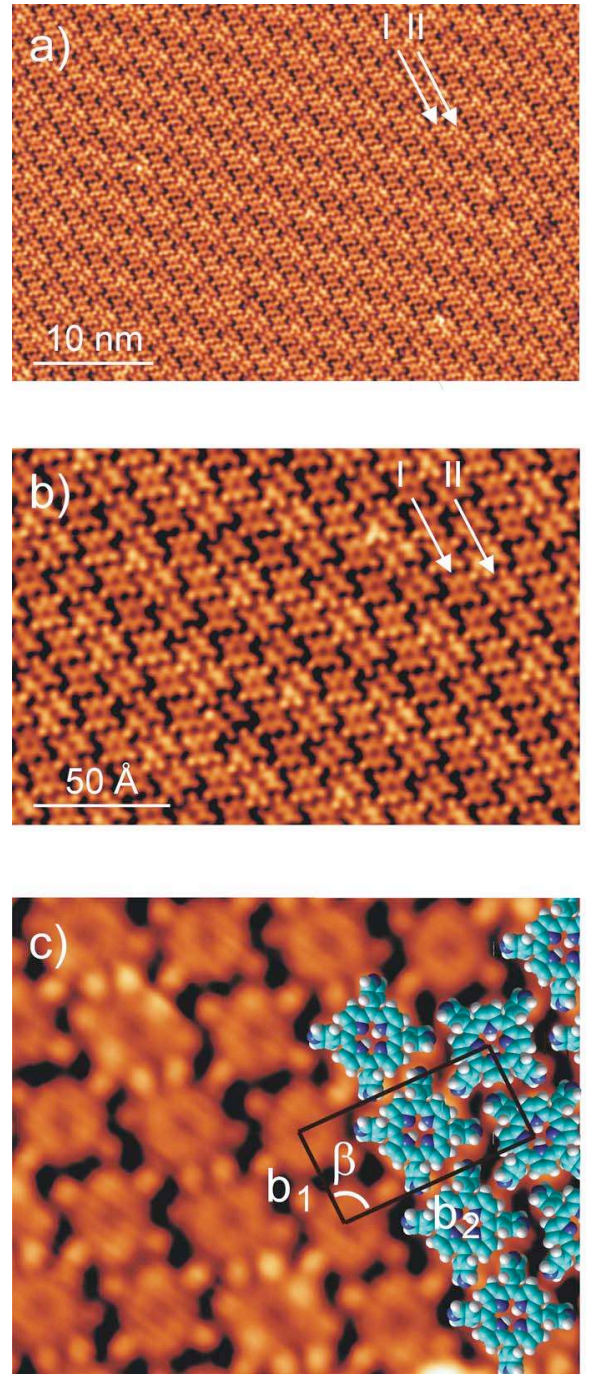

FIG. 2. (Color online) Characteristic structure of self-assembled TPyP layer on $\mathrm{Ag}(111)$. (a) The overview STM image shows that all molecules within a row following the direction marked by the arrows are aligned the same way, while there are two distinguishable rows (I, II) exhibiting a different azimuthal orientation of the molecules $\left(V_{\text {sample }}=-0.3 \mathrm{~V}, I=0.8 \mathrm{nA}\right)$. (b) On an intermediate scale the molecular entities are easily recognizable. (c) The high-resolution image allows to identify intramolecular features $\left(V_{\text {sample }}=\right.$ $-1.2 \mathrm{~V}, I=0.7 \mathrm{nA}$ ). Besides the core with a depression in the center, four protrusions corresponding to the legs are clearly discernible. The TPyP models including the unit cell $\left(b_{1}, b_{2}\right)$ highlight the structure and facilitate the identification of core and terminal pyridyl groups. (see text for details).

mains experience the substrate potential and the azimuthal orientation of the molecular layer is given by the interaction with the substrate. In the experimental data, all possible six distinguishable azimuthal domain orientations $(-\alpha,-\alpha$ $\left.-60^{\circ},-\alpha-120^{\circ} ; \alpha, \alpha+60^{\circ}, \alpha+120^{\circ}\right)$ are observed.

To check the registry of the TPyP overlayer with the subjacent $\operatorname{Ag}(111)$ substrate we evaluate the epitaxy matrix $C$ relating the overlayer unit cell vectors $\left(b_{1}, b_{2}\right)$ to primitive basis vectors of the substrate lattice $\left(a_{1}, a_{2}\right)$. The latter are extracted from atomic resolution images recorded on bare $\operatorname{Ag}(111)$ patches in between TPyP islands.

$$
\left[\begin{array}{l}
b_{1} \\
b_{2}
\end{array}\right]=C\left[\begin{array}{l}
a_{1} \\
a_{2}
\end{array}\right]=\left[\begin{array}{cc}
3.9 & 1.5 \\
-7.5 & 10.7
\end{array}\right]\left[\begin{array}{l}
a_{1} \\
a_{2}
\end{array}\right] .
$$

As no element of the matrix $C$ is integer, ${ }^{45}$ we conclude

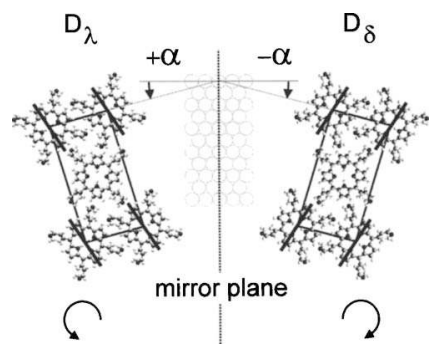

FIG. 3. Model of the two mirror-symmetric domain types observed for TPyP on $\mathrm{Ag}(111)$. The axis of the corner molecules is either rotated clockwise (on the right) or counterclockwise (on the left) away from the long side of the unit cell. Simultaneously the domains are rotated by an angle $\alpha$ of $16^{\circ}$ away from the close-packed substrate rows, one domain clockwise (-), the other counterclockwise $(+)$. Thus, a substrate high-symmetry direction represents a mirror plane for the two domain types, indicating that the azimuthal orientation of the domains is mediated by the interaction with the substrate.

that the TPyP overlayer is not commensurate with the underlying $\operatorname{Ag}(111)$ substrate. This notation based on a classification scheme for molecule-metal heteroepitaxy ${ }^{46,47}$ signals that not every equivalent molecule in the adlayer unit cell resides on an equivalent substrate lattice site. Accordingly, variation in the imaging height exist. Figure 4 shows a longrange intensity modulation, a quasihexagonal Moiré pattern with a periodicity of roughly $43 \AA$ along the molecular rows. This indicates that the lateral intermolecular interactions dominate over site-specific adsorption on the smooth $\operatorname{Ag}(111)$ substrate. The Moiré pattern is most pronounced in STM images recorded at low bias voltages. As the molecules act as scattering centers for the surface state electrons ${ }^{48}$ one observes a standing wave pattern on the bare $\mathrm{Ag}(111)$ region in the bottom part of Fig. 4.

The observed molecular packing is in accordance with the results from elementary molecular mechanics calculations. We applied the MM+ force field of the HYPERCHEM 7.5 molecular modeling package to calculate the noncovalent interactions, i.e., electrostatic and van der Waals forces ${ }^{49}$ as a

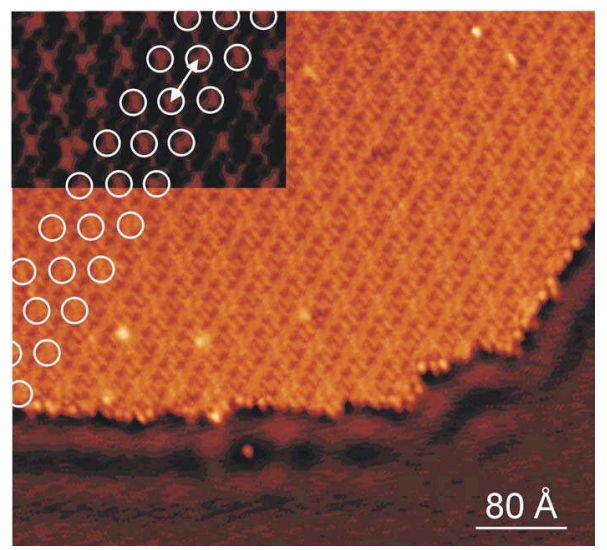

FIG. 4. (Color online) STM image at low sample voltage exhibiting a longrange height modulation, a Moiré pattern. In the top left corner of the image the contrast is increased to emphasize the modulation. The white circles highlight the regions with an increased apparent height. Along the rows these areas are separated by about $43 \AA$ (arrow). In the bottom part of the image the bare $\mathrm{Ag}(111)$ surface is exposed exhibiting the well known standing wave pattern generated by surface state electrons reflected at the molecular lattice $\left(V_{\text {sample }}=-50 \mathrm{mV}, I=0.65 \mathrm{nA}\right)$. 
a)

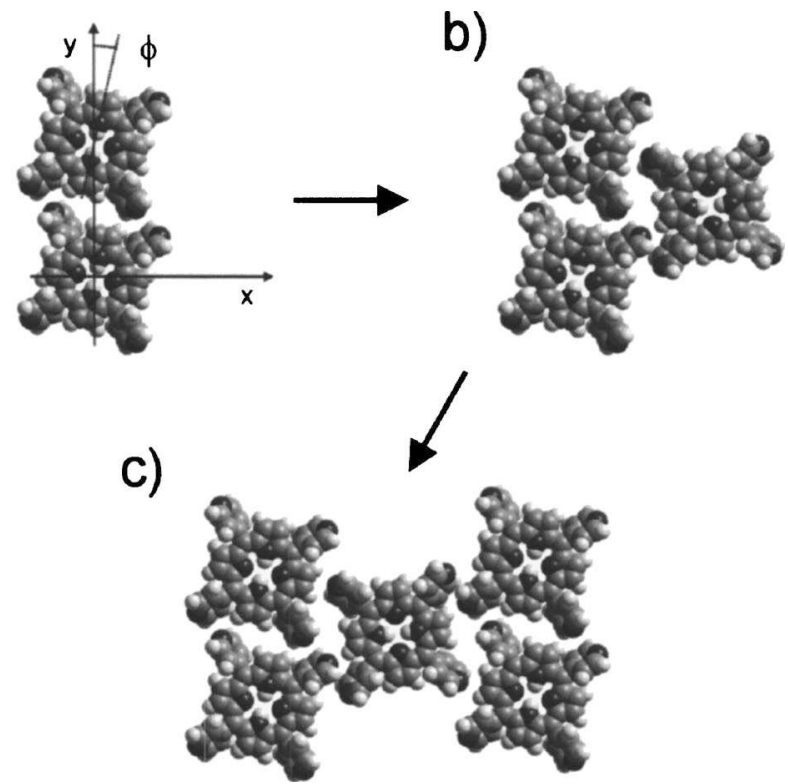

FIG. 5. Lowest energy configurations for (a) two and (b) three TPyP molecules confined to two dimensions, as calculated by applying the MM+ force field of the HYPERCHEM 7.5 package (Ref. 49). (c) shows the complete unit cell structure as extrapolated from (b). The resulting molecular packing scheme is very close to the experimentally observed one (cf. Fig. 2).

function of intermolecular distance and orientation. A similar procedure proved to reflect the relative energetics of different supramolecular arrangements formed by organic molecules on noble metal substrates quite accurately. ${ }^{50,51}$ The very low diffusion barrier and Moiré pattern formation indicate weak site-specific coupling to the $\operatorname{Ag}(111)$ lattice. Thus we neglect electronic interactions with the substrate, the only restriction being that an in-plane alignment of the porphyrin cores is enforced. Starting with two molecules, position $(x, y)$ and orientation $(\phi)$ of the two rigid entities relative to each other are changed in steps of $0.2 \AA(\Delta x, \Delta y)$ and $5^{\circ}(\Delta \phi)$, respectively [cf. Fig. 5(a)]. During this process the geometry of a single molecule is not altered, we rather use the conformation shown in Fig. 1 which is fully consistent with the experimental findings presented in the next section. From all the possible configurations the one shown in Fig. 5(a) has the lowest total energy. It is very close to the in-row alignment observed in the STM data (cf. Fig. 2). This pair formation accounts for an energy gain of about $0.2 \mathrm{eV} /$ molecule. In a next step a third molecule is added to find the best arrangement [Fig. 5(b)]. It is straightforward to extend this structure to the complete unit cell [Fig. 5(c)]. The calculated molecular packing scheme closely resembles the experimentally observed one. One obtains the parallel alignment of the corner molecules, while the central molecule has a different azimuthal orientation. Thus the lateral coupling mediated by the pyridyl groups seems to be decisive for the molecular packing. Accordingly, a phenyl terminated porphyrin species forms a square unit cell on $\mathrm{Ag}(111)$ exhibiting a single molecular orientation. ${ }^{52}$

\section{B. Molecular imaging and conformation}

After discussing the ordering of TPyP on $\operatorname{Ag}(111)$, we now address the imaging of the molecule's intramolecular
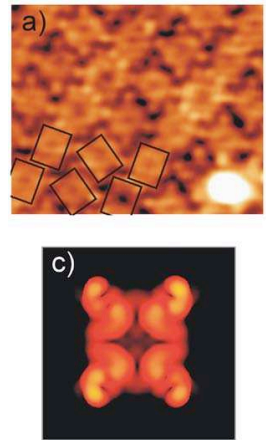
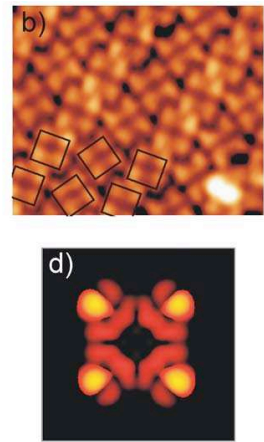

FIG. 6. (Color online) Voltage-dependent imaging of TPyP on $\operatorname{Ag}(111)$. STM images representing (a) the occupied $\left(V_{\text {sample }}=-0.9 \mathrm{~V}, I=0.65 \mathrm{nA}\right)$ and (b) the unoccupied electronic states $\left(V_{\text {sample }}=1.6 \mathrm{~V}, I=0.65 \mathrm{nA}\right)$. Both images display the same surface area, the defect on the bottom right can be used as marker. STM image simulations based on the semiempirical extended Hückel method nicely reflect the rectangular shape for the occupied states (c) and the squarelike pattern dominated by the pyridyl groups for the unoccupied orbitals (d).

features and its conformation. At negative sample bias voltages where the occupied states of the molecule contribute to the tunneling current, the envelope of the molecules defines a rectangle (cf. Fig. 2). The reason for this deviation from a squarelike geometry is that the pyridyl groups are alternately rotated out of the porphyrin plane, as depicted in Fig. 1. ${ }^{8,13}$ This reduces the symmetry from square planar $\left(D_{4 h}\right)$ to $D_{2 d}$ and results in two different side lengths $S_{1}$ and $S_{2}$. By contrast, when we apply high positive sample voltages exceeding $\sim 0.7 \mathrm{~V}$, we probe the unoccupied molecular orbitals and the molecular shape is close to a square. This voltage dependence is clearly visible comparing the STM images in Figs. 6(a) and 6(b), displaying the same surface region. A detailed discussion of the electronic structure of TPyP on $\operatorname{Ag}(111)$ is beyond the scope of this article and will be addressed elsewhere. ${ }^{52}$ However, to interpret the contrast mechanism we simulated the STM images based on semiempirical extended Hückel calculations in the framework of the HYPERCHEM package. ${ }^{51}$ A constant electron density contour is obtained by integrating over the relevant molecular orbitals, which mimics a constant current STM image. ${ }^{53,54}$ The electron density is given by a three-dimensional reconstruction of the Slater orbitals on the basis of the eigenvectors calculated by HYPERCHEM. The vertical position of the contour is displayed in a color-coded image [Figs. 6(c) and 6(d)]. One can vary the density level for which the contour is plotted to get images representing different tip-sample distances and different tunneling currents, respectively. Similar methods relating calculated charge density maps of molecular orbitals to experimental STM data proved to be rather successful. ${ }^{55-58}$ Also in our case, the general molecular appearance can be reproduced surprisingly well, despite ignoring the substrate: for the occupied states we get some intensity from the core and the legs have roughly the same apparent height [Fig. 6(c), contributing molecular orbitals: highest occupied molecular orbital (HOMO) to HOMO-6]. For the unoccupied states the four bright lobes corresponding to the legs dominate the images [Fig. 6(d), contributing molecular orbitals: lowest unoccupied molecular orbital (LUMO) to LUMO+4]. These findings indicate that the mo- 

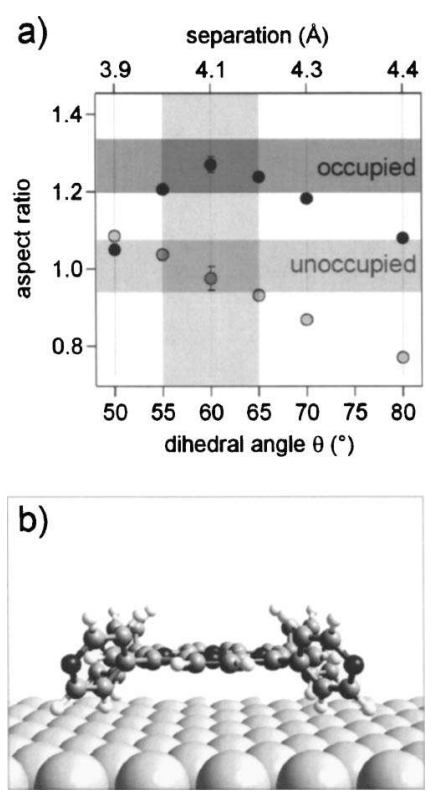

FIG. 7. (a) Comparison of the aspect ratio $\left(=S_{1} / S_{2}\right.$, cf. Fig. 1) extracted from the STM data (horizontal bars) to the aspect ratios from calculated images for different dihedral angles $\Theta$ (circles). This method provides a self-consistency test, since both the aspect ratios for the occupied (dark gray) and unoccupied states (light gray) are measured and calculated independently. Reducing the integration range (by three molecular orbitals) has a clearly smaller impact on the aspect ratios than changing the angle by a $5^{\circ}$ step as indicated by the error bars. The comparison yields $\Theta=60 \pm 5^{\circ}$. This angle is directly related to the separation (about $4 \AA$ ) of the TPyP macrocycle from the outermost $\operatorname{Ag}(111)$ layer as visualized by the perspective view in (b).

lecular orbitals are not too strongly perturbed by the substrate electrons. ${ }^{52}$ Furthermore, the aspect ratio defined as $S_{1} / S_{2}$ depends sensitively on the orientation of the pyridyl legs. Consequently, one can employ these simulations in conjunction with the high-resolution STM data to determine the molecular conformation. To do so, we calculate images for different dihedral angles $\Theta$, and extract the aspect ratios. They are plotted in Fig. 7 as a function of $\Theta$ for the occupied and unoccupied states and compared to the experimental observations. The best agreement is achieved for a dihedral angle of $60 \pm 5^{\circ}$. This value relates well to a TPyP dihedral angle $\Theta$ of $\sim 62^{\circ}$ in a solid state phase, ${ }^{7}$ to $\Theta$ of $63^{\circ}$ determined by $a b$ initio calculations for the ground state of a gas phase TPP molecule ${ }^{13}$ or to $\Theta=59^{\circ}$ for TPP on a $\mathrm{MoS}_{2}$ surface extracted by x-ray absorption spectroscopy. ${ }^{59}$ The similarity of these values for porphyrin molecules in completely different environments might be explained by the onset of energetically unfavorable ruffling of the molecular core due to steric interaction with the rotated mesosubstituents around this $\Theta$ angle. While a significant distortion of the porphyrin core is not consistent with our experimental data, a slight nonplanar deformation cannot be ruled out. It is important to note that $\Theta$ is directly related to the separation of the molecular core from the $\operatorname{Ag}(111)$ substrate (cf. Fig. 7). Thus it controls, for example, the coupling of the metal center in metalloporphyrins to the substrate. ${ }^{39}$ The assumption of a perpendicular orientation of terminal aromatic ring systems, where many reports are based on, might not be well justified.

\section{CONCLUSION}

In summary, highly ordered chiral domains have been observed upon deposition of TPyP on $\operatorname{Ag}(111)$ in a wide temperature range. The characteristic supramolecular structure is explained in terms of intermolecular interactions mediated by the terminal pyridyl groups. The observation of a Moiré pattern points only to a faint influence of the $\operatorname{Ag}(111)$ atomic lattice. A new approach to determine the conformational adaptation of adsorbed species by STM and simple electronic configuration analysis was presented: the dihedral angle $\Theta$ is roughly $60^{\circ}$. This finding is an important feature as the separation of the molecular core from the substrate is an important factor in the functionality of 2-D porphyrin layers.

\section{ACKNOWLEDGMENTS}

The authors acknowledge partial funding by the Canadian CFI, NSERC, and BCKDF programs. One of the authors (W.A.) thanks the Swiss National Science Foundation for financial support, and another author (A.W.-B.) appreciates a scholarship from DAAD.

${ }^{1}$ C. M. Drain, Proc. Natl. Acad. Sci. U.S.A. 99, 5178 (2002).

${ }^{2}$ The Porphyrins, edited by D. Dolphin (Academic, New York, 1979).

${ }^{3}$ S. R. Forrest, Chem. Rev. (Washington, D.C.) 97, 1793 (1997).

${ }^{4}$ R. Paolesse, C. Di Natale, V. Campo Dall'Orto, A. Macagnano, A. Angelaccio, N. Motta, A. Sgarlata, J. Hurst, I. Rezzano, M. Mascini, and A. D'Amico, Thin Solid Films 354, 245 (1999).

${ }^{5}$ L. Pan, S. Kelly, X. Huang, and J. Li, Chem. Commun. (Cambridge) 2002, 2334.

${ }^{6}$ E. B. Fleischer, C. K. Miller, and L. E. Webb, J. Am. Chem. Soc. 86, 2342 (1964).

${ }^{7}$ S. J. Silvers and A. Tulinsky, J. Am. Chem. Soc. 89, 3331 (1967).

${ }^{8}$ T. A. Jung, R. R. Schlittler, and J. K. Gimzewski, Nature (London) 386, 696 (1997).

${ }^{9}$ F. Moresco, G. Meyer, K.-H. Rieder, J. Ping, H. Tang, and C. Joachim, Surf. Sci. 499, 94 (2002).

${ }^{10}$ F. Moresco, G. Meyer, and K.-H. Rieder, Phys. Rev. Lett. 86, 672 (2001).

${ }^{11}$ Ch. Loppacher, M. Guggisberg, O. Pfeiffer, E. Meyer, M. Bammerlin, R. Lüthi, R. Schlitter, J. K. Gimzewski, H. Tang, and C. Joachim, Phys. Rev. Lett. 90, 066107 (2003).

${ }^{12}$ E. B. Fleischer and A. L. Stone, Chem. Commun. (London) 1967, 332.

${ }^{13}$ T. Yokoyama, S. Yokoyama, T. Kamikado, and S. Mashiko, J. Chem. Phys. 115, 3814 (2001).

${ }^{14}$ J. A. Shelnutt, X.-Z. Song, J.-G. Ma, S.-L. Jia, W. Jentzen, and C. J. Medforth, Chem. Soc. Rev. 27, 31 (1998).

${ }^{15}$ M. E. Kosal and K. S. Suslick, J. Solid State Chem. 152, 87 (2000).

${ }^{16}$ S.-B. Lei, C. Wang, S. X. Yin, H. N. Wang, F. Xi, H. W. Liu, B. Xu, L. J. Wan, and C. L. Bai, J. Phys. Chem. B 105, 10838 (2001).

${ }^{17}$ T. Yokoyama, S. Yokoyama, T. Kamikado, Y. Okuno, and S. Mashiko, Nature (London) 413, 619 (2001).

${ }^{18}$ M. Kunitake, N. Batina, and K. Itaya, Langmuir 11, 2337 (1995).

${ }^{19}$ K. Ogaki, N. Batina, M. Kunitake, and K. Itaya, J. Phys. Chem. 100, 7185 (1996).

${ }^{20}$ M. Kunitake, U. Akiba, N. Batina, and K. Itaya, Langmuir 13, 1607 (1997).

${ }^{21}$ L.-J. Wan, S. Shundo, J. Inukai, and K. Itaya, Langmuir 16, 2164 (2000).

${ }^{22}$ Y. He, T. Ye, and E. Borguet, J. Am. Chem. Soc. 124, 11964 (2002).

${ }^{23}$ K. Suto, S. Yoshimoto, and K. Itaya, J. Am. Chem. Soc. 125, 14976 (2003).

${ }^{24}$ T. Ikeda, M. Asakawa, M. Goto, K. Miyake, T. Ishida, and T. Shimizu, Langmuir 20, 5454 (2004).

${ }^{25}$ J. K. Gimzewski, T. A. Jung, M. T. Cuberes, and R. R. Schlittler, Surf. Sci. 386, 101 (1997).

${ }^{26}$ L. Scudiero, D. E. Barlow, U. Mazur, and K. W. Hipps, J. Am. Chem. Soc. 123, 4073 (2001).

${ }^{27}$ H. Suzuki, S. Berner, M. Brunner, H. Yanagi, D. Schlettwein, T. A. Jung, 
and H.-J. Güntherodt, Thin Solid Films 393, 325 (2001).

${ }^{28}$ L. Scudiero, K. W. Hipps, and D. E. Barlow, J. Phys. Chem. B 107, 2903 (2003).

${ }^{29}$ L. Scudiero, D. E. Barlow, and K. W. Hipps, Langmuir 20, 4413 (2004).

${ }^{30}$ D. Bonifazi, H. Spillman, A. Kiebele, M. de Wild, P. Seiler, F. Cheng, H.-J. Günderodt, T. Jung, and F. Diedrich, Angew. Chem., Int. Ed. 43, 4759 (2004)

${ }^{31}$ C. M. Drain and J.-M. Lehn, J. Chem. Soc., Chem. Commun. 1994, 2313.

${ }^{32}$ P. J. Stang, J. Fan, and B. Olenyuk, Chem. Commun. (Cambridge) 1997 , 1453

${ }^{33}$ M. Fujita, Chem. Soc. Rev. 27, 417 (1998).

${ }^{34}$ M. Fujita, Struct. Bonding (Berlin) 96, 177 (2000).

${ }^{35}$ K.-J. Lin, Angew. Chem., Int. Ed. 38, 2730 (1999).

${ }^{36}$ D. Hagrman, P. J. Hagrman, and J. Zubieta, Angew. Chem., Int. Ed. 38 3165 (1999).

${ }^{37}$ G. Meyer, Rev. Sci. Instrum. 67, 2960 (1996).

${ }^{38}$ VTS-CreaTec GmbH, Industriestr. 9, D-74391 Erligheim, Germany, http://www.lt-stm.com/

${ }^{39}$ L. Scudiero, D. E. Barlow, and K. W. Hipps, J. Phys. Chem. B 104, 11899 (2000).

${ }^{40}$ A. Scheybal, T. Ramsvik, R. Bertschinger, M. Putero, F. Nolting, and T. A. Jung, Chem. Phys. Lett. 411, 214 (2005).

${ }^{41}$ W. Deng and K. W. Hipps, J. Phys. Chem. B 107, 10736 (2003).

${ }^{42}$ P. Messina, A. Dmitriev, N. Lin, H. Spillman, M. Abel, J. V. Barth, and K. Kern, J. Am. Chem. Soc. 124, 14000 (2002).

${ }^{43}$ H. Spillmann, A. Dmietriev, N. Lin, P. Messina, J. V. Barth, and K. Kern, J. Am. Chem. Soc. 125, 10725 (2003).

${ }^{44}$ S. M. Barlow and R. Raval, Surf. Sci. Rep. 50, 201 (2003).
${ }^{45}$ The elements in $\mathrm{C}$ are noted only for one specific domain without loss of generality.

${ }^{46}$ R. L. Park and H. H. Madden, Surf. Sci. 11, 188 (1968).

${ }^{47}$ D. E. Hooks, T. Fritz, and M. D. Ward, Adv. Mater. (Weinheim, Ger.) 13, 227 (2001).

${ }^{48}$ L. Gross, F. Moresco, L. Savio, A. Gourdon, C. Joachim, and K. H. Rieder, Phys. Rev. Lett. 93, 056103 (2004).

${ }^{49}$ HYPERCHEM(TM), Hypercube, Inc., 1115 NW 4th Street, Gainesville, Florida 32601; www.hyper.com

${ }^{50}$ J. V. Barth, J. Weckesser, G. Trimarchi, M. Vladimirova, A. De Vita, C. Cai, H. Brune, P. Günter, and K. Kern, J. Am. Chem. Soc. 124, 7991 (2002).

${ }^{51}$ R. Fasel, M. Parschau, and K.-H. Ernst, Angew. Chem., Int. Ed. 42, 5178 (2003).

${ }^{52}$ W. Auwärter, A. Weber, A. Schiffrin, and J. V. Barth (in preparation).

${ }^{53}$ O. Gröning and R. Fasel, STM generator software, EMPA Materials Science and Technology, Switzerland, 2004.

${ }^{54} \mathrm{O}$. Gröning and R. Fasel (to be published).

${ }^{55}$ P. H. Lippel, R. J. Wilson, M. D. Miller, Ch. Wöll, and S. Chiang, Phys. Rev. Lett. 62, 171 (1989).

${ }^{56}$ V. M. Hallmark, S. Chiang, K.-P. Meinhardt, and K. Hafner, Phys. Rev. Lett. 70, 3740 (1993).

${ }^{57}$ P. Sautet, Chem. Rev. (Washington, D.C.) 97, 1097 (1997).

${ }^{58}$ R. Fasel, M. Parschau, and K.-H. Ernst, Nature (London) 439, 449 (2006).

${ }^{59}$ M. P. de Jong, R. Friedlein, S. L. Sorensen, G. Öhrwall, W. Osikowicz, C. Tengsted, S. K. M. Jönsson, M. Fahlman, and W. R. Salaneck, Phys. Rev. B 72, 035448 (2005). 